This item was submitted to Loughborough's Research Repository by the author.

Items in Figshare are protected by copyright, with all rights reserved, unless otherwise indicated.

\title{
Assessment of the critical clearing time in low rotational inertia power systems
}

PLEASE CITE THE PUBLISHED VERSION

https://doi.org/10.1109/SIELA.2018.8447128

PUBLISHER

(C) IEEE

VERSION

AM (Accepted Manuscript)

LICENCE

CC BY-NC-ND 4.0

REPOSITORY RECORD

Gonzalez-Longatt, Francisco M., Jose L. Rueda, and Dimitar Bogdanov. 2019. "Assessment of the Critical Clearing Time in Low Rotational Inertia Power Systems”. figshare. https://hdl.handle.net/2134/33490. 


\section{Assessment of the Critical Clearing Time in Low Rotational Inertia Power Systems}

\author{
F. Gonzalez-Longatt \\ The Wolfson School: Electronic, \\ Electrical, and Systems Engineering \\ Loughborough University \\ Loughborough, UK \\ fglongatt@fglongatt.org
}

\author{
Jose Luis Rueda \\ Delft University of Technology, \\ Engineering and Electrical \\ Engineering, Postbus 52600 AA \\ Delft, Netherlands. Email: \\ J.L.RuedaTorres@tudelft.nl
}

\author{
D. Bogdanov \\ Technical University of Sofia, \\ Faculty of electrical engineering, \\ 18, Kl. Ohridski Blvd. \\ 1000 Sofia, Bulgaria \\ dbogdanov@tu-sofia.bg
}

\begin{abstract}
The growing share of power electronic/converter interfaced generation is decreasing the total system rotational inertia. The reduced rotational inertia in the power system has important impact on the electro-mechanical processes, the power angle and frequency evolution time are quicker resulting in faster transient processes. Rapid response of the protection systems shall be applied, in order to clear the faults in the power systems. This paper aims to identify the roots/mechanism of critical clearing time reduction/deterioration by determining/analyzing the trajectories of the critical clearing time (CCT) in low rotational inertia power systems. In this paper, the equal area criterion (EAC) is used for analysis purposes. Theoretical and practical findings demonstrate the increase of the rotational inertia increases the CCT.
\end{abstract}

Keywords-Angle stability, critical clearing time, low inertia, protection, stability.

\section{INTRODUCTION}

Power system dynamic processes are very complex and system stability is one of the most; they depend on a very large number of factors, including the type of disturbance, predisturbance operating state, network strength, settings and nonlinear response of components[1]. Due to a large number of components involved, each system modelled by some set differential-algebraic equations (DAE), software-based simulation of power systems constitutes a complex computational task. The complexity is mainly caused by the intrinsic non-linearities related to the power system components and their interactions and scale of the mathematical problem (number of components).

Power system stability has been an important research subject for years; there is significant number of research studies dealing with the classical power angle stability [2]-[4]. The transient stability assessment (TSA) is dedicated to analysing the ability of a power system to maintain synchronism when subjected to severe transient disturbance. A traditional transient stability metric for short-circuiting faults on a classical power system is the well-known critical clearing time (CCT).

The CCT has been used as a key metric in the TSA in order to ensure the secure power system operation. The IEEE std 100 [5] defines the clearing time as the time elapsing from the beginning of an overcurrent to the final circuit interruption. The CCT can be easily defined as the maximum time $\left(t_{c}\right)$ during which a disturbance can be applied to the power system without losing the synchronism of the synchronous machines. The growing share of power converter-based technologies, especially those applied in renewable generation systems like wind power and solar photovoltaic (PV) power plants is decreasing the total system rotational inertia. The reduced rotational inertia has an important impact on the electromechanical processes in the power system: the power angle and frequency evolution time are shorter (in the result of the faster transient processes) with low rotational inertia, making the power system control and operation more challenging. Rapid response of the protection systems shall be applied, in order to clear the faults in the power systems.

This paper presents an assessment of the critical clearing time in low rotational inertia power systems. Section II uses a single-machine infinite bus (SMIB) power system to introduce the theoretical background behind the TSA and CCT calculation. Section III presents simulation results of a SMIB and multimachine system to demonstrate the effect of low rotational inertia in the CCT. Section IV concludes and presents the scope of future research.

\section{ROTATIONAL INERTIA AND SYSTEM RESPONSE}

Consider the simple case of an (equivalent) two-machine which consists of a classical synchronous generator $(\mathrm{G})$ directly connected to a hypothetical infinitive bus $\left(H_{\infty}=\infty\right)$, see Fig 1a. Under loss-less steady-state conditions the mechanical rotational speed is equal to the synchronous electrical speed, $\omega(t)=\omega_{\text {syn }}=\omega_{\text {elec }}$ (lossless system), and the phase angle is equal to $\delta_{0}$. Assuming that the generator is operating in steadystate, there is an equilibrium between the input mechanical torque applied to the shaft of the generator $\left(T_{\text {mec }}\right)$ by the primemover and the electro-magnetic torque $\left(T_{\text {elec }}\right)$ output of the generator.

A sudden disturbance, change on the electrical or mechanical torque, breaks the torque balance $\left(T_{\text {net }} \neq 0\right)$, as a consequence, the generator is exposed to the algebraic difference of the accelerating shaft torque and the retarding electromagnetic torque:

$$
T_{\text {net }}=T_{\text {mec }}-T_{\text {elec }}
$$

The laws of rotation apply to the motion of a synchronous generator, and it states that the net torque $\left(T_{n e t}\right)$ is equal to the 
product of angular acceleration $(\alpha)$ and the total moment of inertia of the rotor mass (I, typical units includes $\mathrm{kg} \cdot \mathrm{m}^{2}$ or $\left.\mathrm{J} \cdot \mathrm{s}^{2}\right)$ :

$$
T_{n e t}=I \alpha(t)=I \frac{d \omega(t)}{d t}=I \frac{d^{2} \theta(t)}{d t^{2}}
$$

It is relevant measure the angular position $(\delta)$ of the rotor with respect to a stationary axis and angular velocity $(\omega)$ with respect to a synchronously rotating reference axis (rotating at $\left.\omega_{\text {syn }}\right)$ than with respect to a stationary axis (reference, see Fig. $1 b)$.

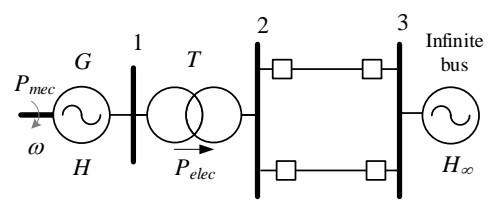

(a)Single-line diagram of a Single-Machine Infinite-Bus(SMIB) Power System.

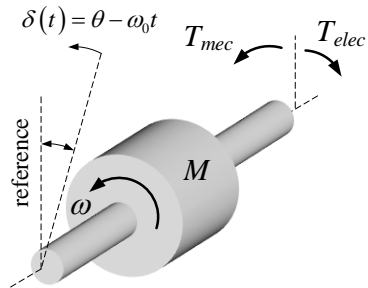

(b) Electromechanical references shown in the spinning rotor.

Figure 1. Realistic SMIB power system and the electro-mechanical reference system at the rotor of the synchronous generator.

The angular position $\delta(t)$ might be defined as:

$$
\delta(t)=\theta-\omega_{\text {syn }} t
$$

where $\omega_{\text {syn }}$ is the rated normal synchronous speed. The first and second derivatives of (2) results:

$$
\begin{aligned}
& \frac{d \delta(t)}{d t}=\frac{d \theta}{d t}-\omega_{0} \\
& \frac{d^{2} \delta(t)}{d t^{2}}=\frac{d^{2} \theta}{d t^{2}}
\end{aligned}
$$

Substituting (5) into (2):

$$
I \frac{d^{2} \delta(t)}{d t^{2}}=T_{n e t}
$$

which is unchanged in form. Now, (1) is introduced into (6):

$$
I \frac{d^{2} \delta(t)}{d t^{2}}=T_{n e t}=T_{\text {mec }}-T_{\text {elec }}
$$

If the above equation is multiplied by the rotational speed $\omega:$

$$
\omega I \frac{d^{2} \delta(t)}{d t^{2}}=\omega I \frac{d \omega(t)}{d t^{2}}=\omega T_{n e t}=\omega T_{\text {mec }}-\omega T_{\text {elec }}
$$

The swing equation is obtained from (8):

$$
M \frac{d^{2} \delta(t)}{d t^{2}}=P_{n e t}=P_{\text {mec }}-P_{\text {elec }}
$$

where the angular momentum of the rotor $(M)$ is calculated as:

$$
M=\omega I
$$

$P_{\text {mec }}=\omega T_{\text {mec }}$ represents the shaft power input, corrected for rotational losses, $P_{\text {elec }}=\omega(2 / P) T_{\text {elec }}$ is the electrical power output, , corrected for electrical losses, $P$ is the poles of the machine and $P_{n e t}$ represents the accelerating power.

Considering the synchronous generator working at nominal condition with a speed $\omega_{s y n}$, the term $\omega d \omega / d t$ in (9) can be expressed through Taylor's expansion evaluated at the nominal condition $\left(\omega_{s y n}\right)$ as:

$$
\begin{gathered}
\omega \frac{d \omega}{d t^{2}}=\left(\omega_{s y n}+\Delta \omega\left(\frac{d \omega / y n}{d t}+\frac{d \Delta \omega}{d t}\right)\right) \\
\omega \frac{d \omega}{d t^{2}}=\omega_{s y n} \frac{d \Delta \omega}{d t}+\Delta \omega \frac{d \Delta \omega}{d t}
\end{gathered}
$$

The term $\Delta \omega d \omega / d t$ contains the product of two small deviations $(\Delta \omega \approx 0$ and $d \omega / d t \approx 0)$ as consequence the term can be easily neglected without significant error.

$$
\begin{aligned}
& \omega \frac{d \omega}{d t^{2}} \approx \omega_{s y n} \frac{d \Delta \omega}{d t} \\
& \omega \frac{d \omega}{d t^{2}}=\omega_{s y n} \frac{d \omega}{d t}
\end{aligned}
$$

The angular momentum $M$ is directly proportional to the rotational speed, as shown in (10), it is evident $M$ is not essentially constant because the speed $\omega$ varies to some extent during the machine swings which follow a disturbance. Considering normal operation of a typical power systems (no affected by low inertia), the changes in the rotor rotational speed $\omega$ is very small when compared to the rated normal synchronous speed and the error introduced by the assumption of angular moment constant is minimum. As a consequence, it has been a tradition, regard the angular momentum as a numerical constant value when solving the swing equation, and equal to $M=\omega_{s y n} I$, sometimes called the angular momentum at normal synchronous speed. The coefficient $I \omega$ in (8) is called the angular momentum of the rotor (rotational version of the linear momentum), when the angular momentum is normalized to the synchronous speed $\omega_{s y n}$, is the inertia constant of the machine $\left(M=\omega_{s y n} I\right)$.

In massive integration of power converter based renewable generation sources, like solar PV and some wind power technologies, is negatively affecting the rotational inertia, it is because the power sources has limited to none possibility of providing real rotational inertia, as a consequence there is a real displacement of traditional thermal generation is leading to a significant reduction in the total rotational system inertia [6]. As a consequence, the power system being less stiff, less resilient to the system frequency disturbances, but also the reduced values of inertia are impacting the whole spectrum of dynamic processes in power systems operation and control [7], [8]. Low inertia scenarios makes the power system dynamic becoming more a more faster as the rotational inertia is reduced [9], [10], as a consequence, the angular momentum more and more variable making a very negative assumption constant value to represent $M$, however, that discussion is beyond the scope of this paper. $M$ is normally expressed in watts per radian per second per second, or joule-seconds per radian. 
The inertia constant $H$ is a constant which has proved very useful, it is defined as the kinetic energy $(K E)$ at rated speed $\left(\omega_{\text {syn }}\right)$ divided by the rated apparent power of the machine $(G$, $M V A)$. There are few interpretations of $H$, but if the units are appropriately selected, $H$ represented the time in seconds a generator can provide rated power solely using the kinetic energy stored in the rotating mass.

$$
H=\frac{K E(\text { stored energy in Joules })}{G(\text { rating in volt-amperes })}
$$

The relationship between the inertia constant $H$ the moment of inertia $M$, is:

$$
G H=M \frac{360 f}{2}
$$

Finally, the swing equations is expressed in terms of the inertia constant.

$$
\frac{H}{\pi f} \frac{d^{2} \delta(t)}{d t^{2}}=P_{\text {net }}=P_{\text {mec }}-P_{\text {elec }}
$$

\section{TRAnSIENT StABILITy AsSESSMENT AND CRITICAL CLEARING TIME}

The dynamic processes, interactions and its correspondent control actions in synchronous-machines-dominated power systems are characterized by a wide spectrum of time constants, where the rotational synchronous inertia defines the main slower electromagnetic phenomena, time scale in order of seconds. However, the dynamic of a low rotational inertia system plays a major role apart of the much slower dynamics, the electro-mechanical variables are volatile in almost every single sense and they are influenced to a much greater extent by the applied control actions.

In this paper, the main interest is the power angle stability that is mainly generator driven, it is related to the electromechanical oscillations between synchronous generators. The power angle instability is encountered in the form of undamped oscillations or a monotonic acceleration of the rotor leading to the loss of synchronism and it tends to have a very fast dynamic, especially in low inertia systems. Transient stability analysis is performed by the use of (i) time domain approach or (ii) direct (Lyapunov-like) methods. In this paper, the equal area criterion (EAC) is used for analysis. The EAC is a powerful graphical application of the Lyapunov's direct method [10]. The EAC is applied to the test system shown in Fig. 1.a. the accelerating $\left(A_{1}\right)$ and accelerating $\left(A_{2}\right)$ areas are shown in Fig. 2.

The equilibrium energy of the electro-mechanical process is defined by the following integral equation (where $\delta_{\max }=\pi-\delta_{0}$ ):

$$
\int_{\delta_{0}}^{\delta_{c}} P_{m e c}^{0} d \delta=\int_{\delta_{c}}^{\delta_{\max }}\left(P_{\max } \sin \delta-P_{m e c}^{0}\right) d \delta
$$

The analytical solution of (15) provides a non-linear equation where the angle $\delta_{c}$ is the variable of interest. The boundary of the stability limit is defined by the critical clearing angle $(\mathrm{CCA}), \delta_{c}$ calculated from the following nonlinear equations:

$$
P_{\text {mec }}^{0}\left(\delta_{c}-\delta_{0}\right)=P_{\text {mec }}^{0}\left(\delta_{0}+\delta_{c}-\pi\right)+P_{\max }\left(\cos \delta_{0}+\cos \delta_{c}\right)
$$

$$
\pi P_{m}^{0}-P_{\max } \cos \left(\delta_{0}\right)-P_{\max } \cos \left(\delta_{c}\right)-2 \delta_{0} P_{m}^{0}=0
$$

Extracting the CCA $\delta_{c}$ from (16):

$$
\begin{aligned}
& \delta_{c}=\cos ^{-1}\left(\frac{P_{m}^{0}\left(\pi-2 \delta_{0}\right)-P_{\max } \cos \left(\delta_{0}\right)}{P_{\max }}\right) \\
& \delta_{c}=\cos ^{-1}\left(\frac{P_{\max } \sin \left(\delta_{0}\right)\left(\pi-2 \delta_{0}\right)-P_{\max } \cos \left(\delta_{0}\right)}{P_{\max }}\right) \\
& \delta_{c}=\cos ^{-1}\left[\left(\pi-2 \delta_{0}\right) \sin \left(\delta_{0}\right)-\cos \left(\delta_{0}\right)\right] \\
& P_{\text {max }} P_{\text {elec }}(\delta)=\frac{\left|E_{G}\right|\left|V_{\infty}\right|}{X_{G \infty}} \sin \delta \\
& \underset{0}{\stackrel{0}{r}}
\end{aligned}
$$

Figure 2. Power-angle diagram for analysing transient disturbances due to faults with subsequent fault cleared.

From (17) is clear the CCA is not depending on the rotational inertia, Fig. 3 shows how the CCA changes as the initial operational state, $\delta_{0}=\sin ^{-1}\left(P^{0}{ }_{\text {med }} / P_{\text {max }}\right)$ is changed by the initial mechanical power $\left(P^{0}{ }_{m e c}\right)$.

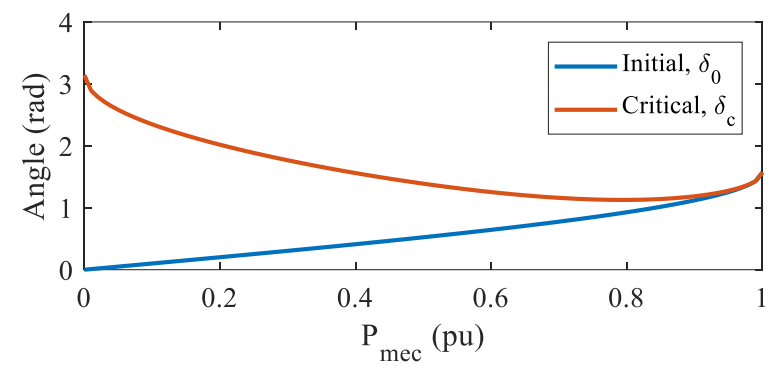

Figure 3. Effect of the initial mechanical power $\left(P^{0}{ }_{\text {mec }}\right)$ in the initial angle $\left(\delta_{0}\right)$ and the CCA $\left(\delta_{\mathrm{c}}\right)$.

Now, assuming there is not intervention of the governor controller, the critical clearing time $\left(t_{c}\right)$ can be obtained by the integration of (14) during the acceleration period:

$$
\begin{gathered}
\frac{H}{\pi f} \frac{d^{2} \delta(t)}{d t^{2}}=P_{\text {mec }}^{0} \quad \rightarrow \quad \frac{d^{2} \delta(t)}{d t^{2}}=\frac{\pi f}{H} P_{\text {mec }}^{0} \\
\frac{d \delta(t)}{d t}=\int_{t=0}^{t} \frac{\pi f}{H} P_{\text {mec }}^{0} d t=\frac{\pi f}{H} P_{\text {mec }}^{0} t
\end{gathered}
$$

Finally, $t_{c}$ is calculated as:

$$
\begin{gathered}
\int_{\delta_{0}}^{\delta_{c}} d \delta=\int_{t=0}^{t} \frac{\pi f}{H} P_{\text {mec }}^{0} t \cdot d t \rightarrow \delta_{c}-\delta_{0}=\frac{\pi f}{H} P_{\text {mec }}^{0}\left(\frac{t_{c}^{2}}{2}\right) \\
t_{c}=\sqrt{\frac{2 H\left(\delta_{c}-\delta_{0}\right)}{\pi f P_{\max } \sin \delta_{0}}}
\end{gathered}
$$

The CCT (18) and CCA (17) are typically used as indicators to measure the power angle transient stability of a power system. Now, from (18) is clear that rotational inertia $(H)$ has effect on the CCT of the synchronous generator of the 
the SMIB. Figure 4 shows the effect of the rotational inertia (H) in the CCT. As predicted by (18) the increase of the rotational inertia increases the CCT.

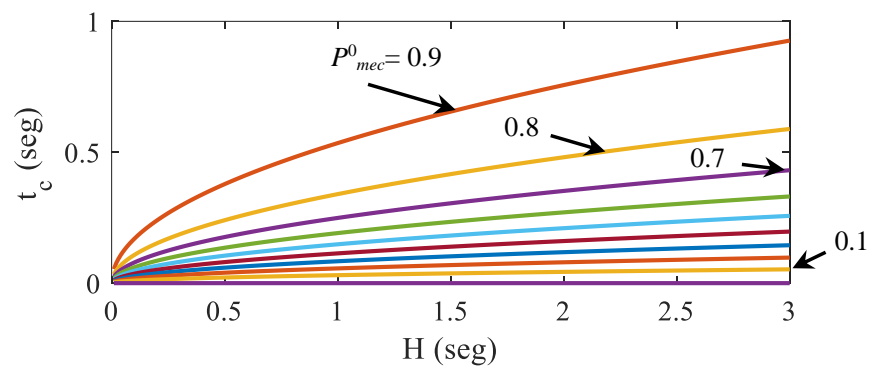

Figure 4. Effect of the rotational inertia $(H)$ and the initial mechanical power on the CCT $\left(t_{\mathrm{c}}\right)$

Finally, a real single machine equivalent system (see Fig. 5) is used to demonstrate the effect of reducing the system inertia on the transient stability. Two illustrative examples of stable and unstable trajectories, considering high and low inertia, are shown in Fig. 6.

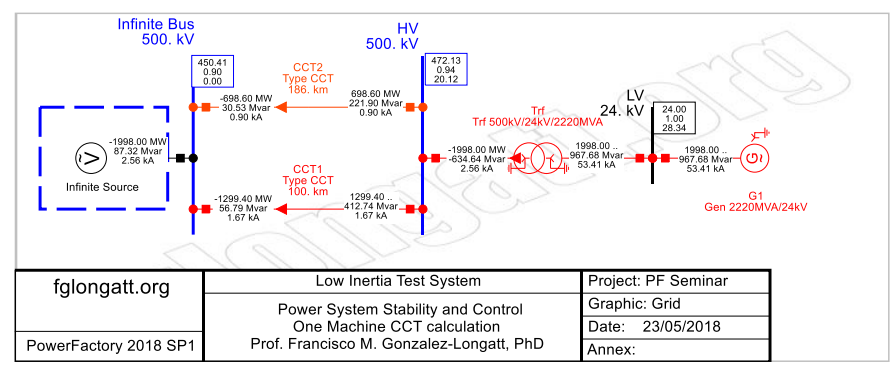

Figure 5. Real SMIB power system implemented in DIgSILENT PowerFactory.

\section{CONCLUSIONS}

Rotational inertia is decreasing in the modern power system, and it is expected to be significantly decreased in the future. Reduced rotational inertia makes the power system electromechanical dynamic of more volatile. This paper presents results of the assessment of the critical clearing time in low rotational inertia power systems. Initially, the assessment has been performed using analytical equations over a SMIB model; then the concepts are demonstrated in multi-machine systems using time-domain simulations. The proposed approach can be applied for the analysis of the power system stability in respect of protection system response time. The application of Phasor Measurement Units (PMUs) can help recognize zones of the power grid in non-stable operational state and respectively initiate tripping of faulty grid branches.

\section{REFERENCES}

[1] F. Gonzalez-Longatt, E. Chikuni, and E. Rashayi, "Effects of the Synthetic Inertia from wind power on the total system inertia after a frequency disturbance," in Proceedings of the IEEE International Conference on Industrial Technology, 2013.

[2] E. W. Kimbark, Power system stability. Vol.3, Synchronous machines. Wiley;Chapman \& Hall, 1956.
[3] P. Kundur, N. J. Balu, and M. G. Lauby, Power system stability and control. New York; London: McGraw-Hill, 1994.

[4] P. M. Anderson, Analysis of faulted power systems. Ames, Iowa: Iowa State University Press, 1991.

[5] S. Edition, "The Authoritative Dictionary of IEEE Standards Terms," IEEE Std 100-2000, pp. 1-1362, 2000.

[6] P. M. Ashton, "Exploiting Phasor Measurement Units for Enhanced Transmission Network Operation and Control," 2014.

[7] F. Gonzalez-Longatt, "Effects of Fast Acting Power Controller of BESS in the System Frequency Response of a Multi-Machine System: -+Grid Technologies (ISGT Asia 2018), 2018.

[8] F. Gonzalez-Longatt, J. Rueda, and E. Vázquez Martínez, "Effect of Fast Acting Power Controller of Battery Energy Storage Systems in the Under-frequency Load Shedding Scheme," in nternational Conference on Innovative Smart Grid Technologies (ISGT Asia 2018), 2018.

[9] A. Ulbig, T. S. Borsche, and G. Andersson, "Analyzing Rotational Inertia, Grid Topology and their Role for Power System Stability," IFACPapersOnLine, vol. 48, no. 30, pp. 541-547, Jan. 2015.

[10]C. Brivio, S. Mandelli, and M. Merlo, "Battery energy storage system for primary control reserve and energy arbitrage," Sustain. Energy, Grids Networks, vol. 6, pp. 152-165, 2016.
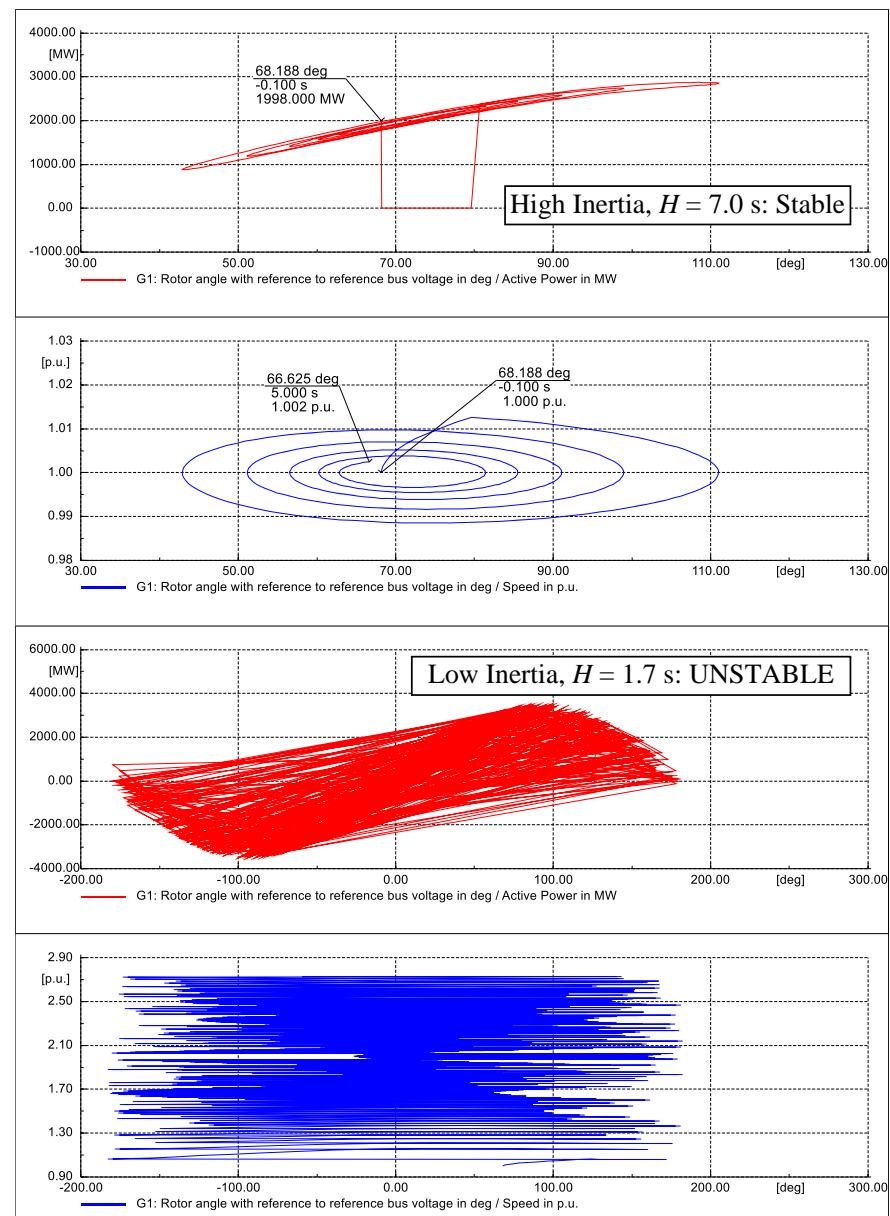

Figure 6. Illustrative trajectories of high and low inertia systems. $P-\delta$ and $\omega$ $\delta$. Clearing time $=100 \mathrm{~ms}$. 